LETTER

Evolution of the universe driven by a mass-

Recent citations dimension-one fermion field

To cite this article: S. H. Pereira et al 2017 EPL 12031001

View the article online for updates and enhancements. 


\title{
Evolution of the universe driven by a mass-dimension-one fermion field
}

\author{
S. H. Pereira ${ }^{1}$, R. F. L. Holanda ${ }^{2,3}$ and A. Pinho S. SouzA ${ }^{1}$ \\ 1 Universidade Estadual Paulista (Unesp) - Faculdade de Engenharia, Guaratinguetá, \\ Departamento de Física e Química - 12516-410, Guaratinguetá, SP, Brazil \\ 2 Universidade Federal de Sergipe, Departamento de Física - 49100-000, Aracaju, SE, Brazil \\ 3 Universidade Federal de Campina Grande, Departamento de Física - 58429-900, Campina Grande, PB, Brazil
}

received 12 December 2017; accepted in final form 10 January 2018

published online 29 January 2018

PACS 12.60.-i - Models beyond the standard model

PACS 98.80.Cq - Particle-theory and field-theory models of the early Universe (including cosmic pancakes, cosmic strings, chaotic phenomena, inflationary universe, etc.)

\begin{abstract}
This paper studies the evolution of the universe filled with a neutral mass-dimensionone fermionic field, sometimes called Elko. The numerical analysis of the coupled system of equations furnishes a scale factor growth and energy density evolution that correctly reproduce the inflationary phase of the universe. After that, assuming a mechanism of energy transference to ordinary matter, the initial conditions generated after inflation drives the radiation-dominated phase and also the subsequent dark-matter evolution, since the Elko field is a good dark-matter candidate. The energy density of the field at the end of inflation, at the end of the radiation phase and for the present time are in agreement with the standard model estimates. The analysis was performed with a potential containing a quadratic mass term plus a quartic self-interaction term, which follows naturally from the theory of mass-dimension-one fermions. Inflation occurs when the field makes a kind of transition around the Planck mass scale. The number of e-foldings during inflation was found to be strongly dependent on the initial conditions of the Elko field, as occurs in chaotic inflationary models. An upper limit to the Elko mass is estimated. A possible interpretation of the inflation as a consequence of a kind of Pauli exclusion principle is presented at the end.
\end{abstract}

Copyright (c) EPLA, 2018

Introduction. - The search for a model that correctly describes the whole evolution of the universe is an old problem in cosmology. In the current model, the universe starts in a very hot and dense phase known as Big Bang ${ }^{1}$ driven by quantum effects based on models as supersymmetry, supergravity, extra dimensions, superstrings, among others. The quantum effects are dominant while the energy density is greater than the Planck energy density $m_{p l}^{4}$, characterized by the Planck mass $m_{p l} \simeq 1.22 \times 10^{19} \mathrm{GeV}$. The age of the universe is about $10^{-43} \mathrm{~s}$ at this stage. From $10^{-43} \mathrm{~s}$ to about $10^{-35} \mathrm{~s}$ the universe is still expanding and cooling in the so-called pre-inflationary phase, its temperature is about $10^{27} \mathrm{~K}$ and the energy density is about $10^{58} \mathrm{GeV}^{4} \sim 10^{75} \mathrm{~g} / \mathrm{cm}^{3}$. From $10^{-35} \mathrm{~s}$ to $10^{-32} \mathrm{~s}$ the

\footnotetext{
${ }^{1}$ See [1] or [2] for a brief resume of the thermal history of the universe.
}

universe undergoes the so-called cosmic inflation, where the scale factor $a(t)$ grows by about $10^{43}$ orders of magnitude. Such phase is necessary in order to solve some problems as the flatness problem, the monopole and relics problems, the horizon problem and homogeneity [3,4]. After such very abrupt expansion, nearly exponential, the energy density is about $10^{51} \mathrm{GeV}^{4} \sim 10^{68} \mathrm{~g} / \mathrm{cm}^{3}$ and the universe goes through a reheating phase, where the socalled inflaton field transfers energy to the ordinary matter, which starts to dominate. After $10^{-32} \mathrm{~s}$ the universe goes into a radiation-dominated phase up to $10^{11} \mathrm{~s}$, and several processes occur, such as the end of electroweak unification, the quark-hadrons transition and nucleosynthesis of light elements. At the end of the radiation-dominated phase, radiation and matter have the same energy density, of about $10^{-34} \mathrm{GeV}^{4} \sim 10^{-17} \mathrm{~g} / \mathrm{cm}^{3}$. Then the universe enters a matter-dominated long epoch, in which occurs the formation of atoms and of the first structures, such 
as galaxies and clusters of galaxies. The scale factor has grown about $10^{65}-10^{75}$ orders of magnitude up today, with an age of about $10^{18} \mathrm{~s}$ and the energy density decreases to about $10^{-46} \mathrm{GeV}^{4} \sim 10^{-29} \mathrm{~g} / \mathrm{cm}^{3}$. Finally, very recently the universe has started a new accelerating phase, dominated by a cosmological constant term or a dark-energy fluid. This is a very brief history of the universe.

An unified model that could describe all the phases of evolution of the universe is a difficult task. The several orders of magnitude involved from the inflation to recent cosmic acceleration forced the researches to divide the evolution of the universe into different parts, each one characterized by different kinds of particles that dominate at different stages. These ingredients form the so-called standard model of cosmology. The inflationary phase of the universe can be constructed with a single scalar field which drives the inflation while the scalar field rolls down to the bottom of its potential. Several potentials that satisfy the fine tunings of the inflationary phase have been studied in the last decades (see [3,4] for a review and [5] for observational constraints on several potentials). After inflation, the scalar field ends in a rapid oscillation around the minimum of its potential and its energy is transferred to the baryonic particles in the next phase of evolution, in a process known as reheating. The scalar field does not act anymore and the universe evolves being dominated by radiation and subsequently by matter. During the matterdominated phase there is the necessity of adding a new kind of non-baryonic matter to the model in order to correctly explain structure formation, thus the standard model needs addition of dark matter, about $25 \%$ of the total content of the universe. Radiation and baryons correspond just to about $5 \%$ of the total content. After the evolution dominated by dark matter, observations show that a recent accelerated phase of expansion needs a new ingredient, the so-called cosmological constant or even a new kind of energy, called dark energy, acting as a vacuum energy density and representing about $70 \%$ of the total energetic content.

As previously stated, a model describing all phases of the evolution of the universe is a challenge for the present cosmology. In particular, the constraints in the energy density and the "size" of the universe after each phase is strongly dependent on the inflationary model adopted. The exact initial conditions leading a cosmic inflation and its connection to radiation and matter evolution depends on the dynamic of the inflaton field. A new class of fermions with mass dimension one named Elko [6-9] was proposed and established in solid quantum bases very recently $[9,10]$ (see also [11]). Such new fermionic field is a natural candidate for dark matter in the universe once it is constructed by means of half-integer spinors that are eigenstates of the charge conjugation operator, being neutral and weakly coupled to the electromagnetic sector of the standard model of particles [12]. Several cosmological applications of such field have been recently presented in the literature [13-23]. The great advantage to use the Elko field as the inflaton field is that it does not need to disappear after acting, since it happens to be responsible for the dark matter in the present days.

In this paper we take the Elko field as responsible for the inflationary phase and if we suppose that it transfers only part of its energy to radiation after inflation ${ }^{2}$, the evolution of universe up to the present time can be correctly described, namely the end of inflation serves as initial condition for the radiation phase and the end of radiation as initial condition for the matter-dominated phase. We present the numerical results concerning the Elko field driving the inflationary phase in the presence of a potential with a quadratic mass term and a quartic self-interaction term. It is shown that inflation occurs when the Elko field makes a transition characterized by a value of about one Planck mass. The Planck scale represents a limit from the pre-inflationary phase to the postinflationary one. The evolution for the scale factor and the energy density during and after a cosmic inflation is in good agreement with the values of the standard model for three different epochs, namely the end of inflation, the transition from radiation to matter dominance and the present-day value for such parameters. It is important to stress that the time scale in seconds used here allows to estimate the real growth of the scale factor and the decrease of the energy density up today. A similar treatment has been done recently in [23] concerning the numerical analysis at the inflationary phase, however, by using a phenomenological symmetry-broken potential, which is not predicted by mass-dimension-one fermions theory [7]. Also, in [23] the evolution after inflation was just extrapolated, not considering the radiation and matter evolution from its known evolution equations, as done here.

Dynamic equations for the Elko field. - In a flat space-time there are four Elko spinors satisfying invariance by the charge conjugation operator $C$, they are labeled as $\lambda_{\beta}^{S / A}$, where $S$ stands for self-conjugate and $A$ for anti-selfconjugate. The index $\beta$ stands for two possible helicities. They satisfy $C \lambda_{\beta}^{S / A}= \pm \lambda_{\beta}^{S / A}[6-11]$ and are normalized satisfying the relation $\vec{\lambda}_{\beta}(\mathbf{k})^{S / A} \lambda_{\beta^{\prime}}(\mathbf{k})^{S / A}= \pm 2 m \delta_{\beta \beta^{\prime}}$, where $\lambda_{\beta^{\prime}}(\mathbf{k})^{S / A}$ and $\vec{\lambda}_{\beta}(\mathbf{k})^{S / A}$ are the usual spinor and its dual, respectively. The dual has been redefined recently [9-11] in order to maintain locality and Lorentz covariance. The corresponding quantum fields can be redefined in order to satisfy a normalization relation as $\vec{\lambda} \lambda= \pm 1$, where $\lambda$ stands here for any of the four spinors, with normalization +1 for the two self-conjugates and -1 for the two anti-self-conjugates.

In this paper we have used just one fermionic Elko field satisfying a positive normalization. Also, in order to use the Elko field in a curved background, we have factored out

\footnotetext{
${ }^{2}$ The mechanism of such transfer is not known yet, but the possible coupling of the Elko field to the Higgs field has been studied recently $[7,24,25]$, thus the Higgs field could be responsible for intermediate such energy transfer to ordinary matter.
} 
the time dependence of the Elko field as [14-23] $\Lambda=\phi(t) \lambda$. The action for the model reads $[18,19]$

$$
S=\int \mathrm{d}^{4} x \sqrt{-g}\left[-\frac{\tilde{R}}{2 \kappa^{2}}+\frac{1}{2} g^{\mu \nu} \tilde{\nabla}_{\mu} \vec{\Lambda} \tilde{\nabla}_{\nu} \Lambda-V(\widehat{\Lambda} \Lambda)\right],
$$

where $\kappa^{2} \equiv 8 \pi G$ with $c=1$. The tilde denotes the presence of torsion terms into the Ricci scalar $\tilde{R}$ and covariant derivatives, namely, $\tilde{\nabla}_{\mu} \Lambda \equiv \partial_{\mu} \Lambda-\Gamma_{\mu} \Lambda$ and $\tilde{\nabla}_{\mu} \vec{\Lambda} \equiv \partial_{\mu} \vec{\Lambda}+\widehat{\Lambda} \Gamma_{\mu}$, where $\Gamma_{\mu}$ is the connection associated to spinor fields, containing the spin connections. For the potential we have used a quadratic mass term and a quartic self-interacting term, namely,

$$
V=\frac{1}{2} m^{2} \vec{\Lambda} \Lambda+\frac{\alpha}{4}(\widehat{\Lambda} \Lambda)^{2}=\frac{1}{2} m^{2} \phi^{2}+\frac{\alpha}{4} \phi^{4},
$$

where $m$ is the physical mass of the field and $\alpha$ a dimensionless coupling. Such kind of potential follows naturally from the theory of mass-dimension-one fermions [7].

In a flat Friedmann-Robertson-Walker (FRW) metric $\mathrm{d} s^{2}=\mathrm{d} t^{2}-a(t)^{2}\left[\mathrm{~d} x^{2}+\mathrm{d} y^{2}+\mathrm{d} z^{2}\right]$, the two Friedmann equations and the dynamic field equation for the time component $\phi(t)$ of the Elko field ${ }^{3}$ plus an ordinary matter $i$ can be obtained $[18,19]$ :

$$
\begin{aligned}
& H^{2}=\frac{\kappa^{2}}{3}\left(1+\frac{\kappa^{2} \phi^{2}}{8}\right)\left[\frac{\dot{\phi}^{2}}{2}+V(\phi)+\rho_{i}\right] \\
& \dot{H}=-\frac{\kappa^{2}}{2}\left(1+\frac{\kappa^{2} \phi^{2}}{8}\right)\left[\dot{\phi}^{2}-\frac{1}{2} \frac{H \phi \dot{\phi}}{\left(1+\kappa^{2} \phi^{2} / 8\right)^{2}}+\rho_{i}+p_{i}\right]
\end{aligned}
$$$$
\ddot{\phi}+3 H \dot{\phi}+\frac{\mathrm{d} V(\phi)}{\mathrm{d} \phi}-\frac{3}{4} \frac{H^{2} \phi}{\left(1+\kappa^{2} \phi^{2} / 8\right)^{2}}=0
$$

where $H=\frac{\dot{a}}{a}$ is the Hubble parameter and the dot stands for time derivative. The energy density $\rho_{i}$ and the pressure $p_{i}$ for the ordinary matter $i$ (radiation or baryonic matter) were also included in the right side of (3) and (4). The equations for energy density and pressure for the Elko field are [19]

$$
\begin{aligned}
\rho= & \frac{\dot{\phi}^{2}}{2}+V(\phi)+\frac{3}{8} \frac{H^{2} \phi^{2}}{\left(1+\kappa^{2} \phi^{2} / 8\right)}, \\
p= & \frac{\dot{\phi}^{2}}{2}-V(\phi)-\frac{3}{8} \frac{H^{2} \phi^{2}}{\left(1+\kappa^{2} \phi^{2} / 8\right)}-\frac{1}{4} \frac{\dot{H} \phi^{2}}{\left(1+\kappa^{2} \phi^{2} / 8\right)} \\
& -\frac{1}{2} \frac{H \phi \dot{\phi}}{\left(1+\kappa^{2} \phi^{2} / 8\right)^{2}} .
\end{aligned}
$$

Such equations generalise those for a standard scalar field. The second terms inside the curly brackets of (3), (4), the second term inside the square brackets of (4), the last terms of (5) and (6) and last three terms of (7) are not present in the standard scalar field equations.

\footnotetext{
${ }^{3}$ We will refer to $\phi(t)$ as Elko field from now on, but the complete spinor structure is present in $\Lambda$.
}

This shows that the Elko dynamic field equations are much richer than the scalar field ones. Such additional terms come from the fact that the fermionic Elko field must be coupled to torsion in an Einstein-Cartan framework and also due to spin connections terms. In particular, since $\kappa^{2}=8 \pi G=8 \pi / m_{p l}^{2}$, in the limit $\phi \ll m_{p l}$ and $H \phi \ll \dot{\phi}$ these additional terms can be discarded and the equations are exactly like those for the standard scalar field. Thus, the limit $\phi \sim m_{p l}$ represents a kind of transition of the Elko field from a high-energy regime dominated by torsion and spin connection terms to a low-energy regime free of torsion and spin connection. Surprisingly, it has been found numerically that such transition from a highenergy regime to a low-energy one is responsible for the inflationary phase of the universe.

Given a potential $V(\phi)$ the above set of equations can be integrated (at least numerically) in order to study the evolution of $a(t)$ and $\phi(t)$. In the following it will set the initial conditions and values of the parameters in order to make a numerical analysis of the system.

Numerical results. - In order to perform a numerical analysis ${ }^{4}$ for the evolution of the scale factor $a(t)$ and of the field $\phi(t)$ we chose to work with the coupled equations (3) and (5). It is also necessary to fix some parameters of the potential (2) and initial conditions for $\phi$. Since (3) is a first-order differential equation in $a(t)$ it needs just one initial condition at $t_{i}$, and it was chosen $a\left(t_{i}\right)=10^{-70}$. With such choice we expect the present value to be $a=1$. Equation (5) is a second-order differential equation and needs two initial conditions. In order to test the hypothesis that inflation occurs due to a transition of the Elko field from a high-energy scale $\phi>m_{p l}$ to a low-energy regime $\phi<m_{p l}$, it was chosen $\phi\left(t_{i}\right) \equiv \phi_{i}=1.75 m_{p l}=3.25 \times 10^{43} \mathrm{~s}^{-1}$, similarly to the chaotic inflationary model by Linde [26]. The inflation must occur when $\phi(t)$ decays from $\phi_{i}$ to zero, at the bottom of the potential. It has been also considered that before inflation occurs the universe was filled with a nearly homogeneous and isotropic gas of Elko particles, nearly at rest, so it was chosen $\dot{\phi}\left(t_{i}\right) \equiv \dot{\phi}_{i}=0$. Thus, the unique energy present at the beginning is its potential energy $V\left(\phi_{i}\right)$. During inflation we do not consider the presence of ordinary matter.

After some numerical analysis it was found that the beginning of the evolution, namely the phase that includes the inflation, can be driven just by the self-interacting quartic term of the potential if $m^{2} \ll \frac{1}{2} \alpha \phi_{i}^{2}$, which is obvious since that it is proportional to $\phi^{4}$ while the mass term is proportional to $\phi^{2}$ and the initial condition for $\phi$ is nearly greater than the Planck mass. By choosing $\alpha=4 \times 10^{-20}$ the condition for the mass is $m \ll 2.5 \times$ $10^{-10} m_{p l} \simeq 3 \times 10^{9} \mathrm{GeV}$. We chose $m=1.0 \mathrm{GeV}$ so that it does not contribute to the evolution at the beginning.

\footnotetext{
${ }^{4}$ It was used the Maple 15 Software, whose numerical solutions are found by a Fehlberg fourth-fifth order Runge-Kutta method with degree four of interpolation.
} 


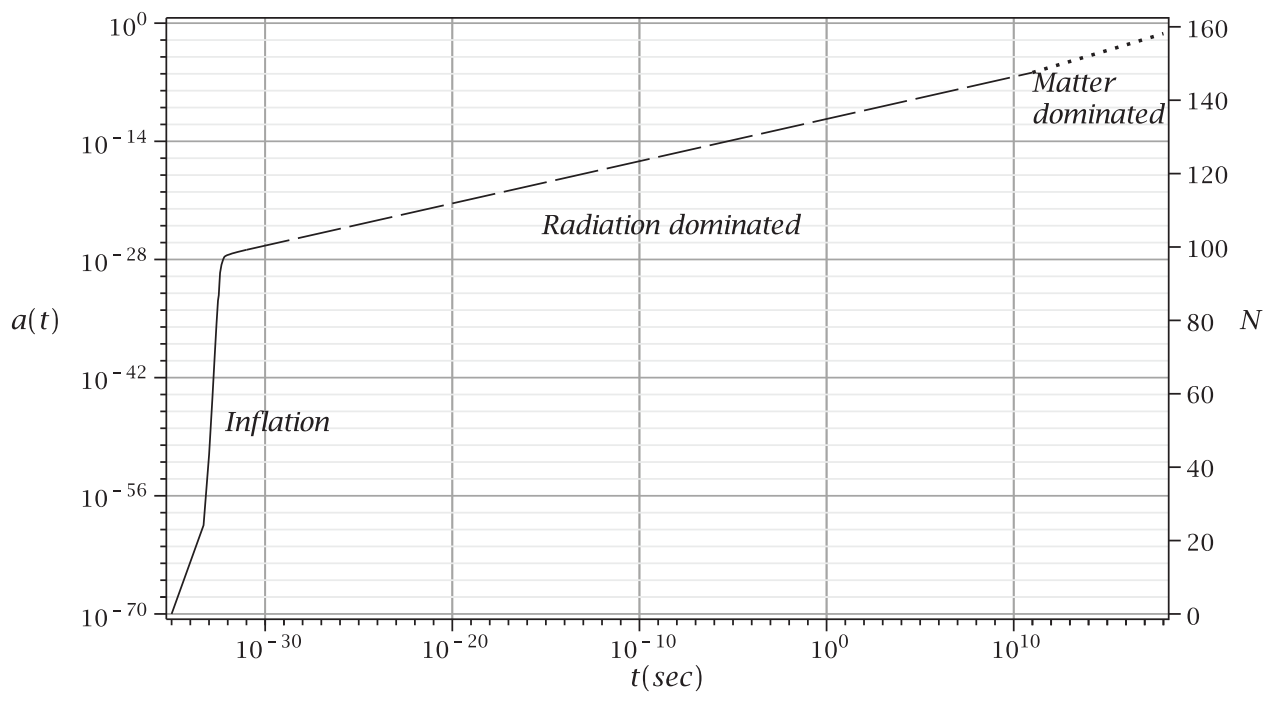

Fig. 1: Numerical result for the evolution of the scale factor $a(t)$ with time. Inflation occurs from $10^{-35} \mathrm{~s}$ to $10^{-32} \mathrm{~s}(\mathrm{solid}$ line) with the scale factor increasing from $10^{-70}$ up to about $10^{-28}$. After that, the scale factor grows smoothly dominated by radiation (dashed line), with $a(t) \sim t^{1 / 2}$ up to about $t \simeq 10^{11} \mathrm{~s}$. From $10^{11} \mathrm{~s}$ to $10^{18} \mathrm{~s}$ the evolution is of matter type (dotted line), with $a(t) \sim t^{2 / 3}$. The growth of the scale factor up to present days, $a(t) \sim 1$, corresponds to an increase of about $10^{70}$. The vertical axis on the right shows the number $N$ of e-foldings.

When the field decays below about $10^{-10} m_{p l} \simeq 10^{33} \mathrm{~s}^{-1}$ the quadratic mass term starts to dominate. Numerically this occurs at about $10^{-5} \mathrm{~s}$, being important already in the radiation-dominated epoch. Having stated the initial conditions and the value of the parameters, we see that the condition $V\left(\phi_{i}\right) \ll m_{p l}^{4}$ is satisfied in order to guarantee that the total energy of the field is below the Planck scale, so that quantum effects are not present. Such condition is satisfied due to the tiny value of $\alpha$. Now it is possible to make a numerical analysis of the system of equations (3) and (5). In order to have the time scale in seconds, we used $\kappa=\sqrt{8 \pi} / m_{p l}=4.1 \times 10^{-19} \mathrm{GeV}^{-1}=2.7 \times 10^{-43} \mathrm{~s}$. The initial time $t_{i}$ was chosen as $t_{i}=10^{-35} \mathrm{~s}$. It is expected that inflation occurs up to about $10^{-32} \mathrm{~s}$ and after that the universe expands nearly as a power law in time.

Figures 1 and 2 present the main results of the paper. In fig. 1 the evolution of the scale factor $a(t)$ with time (in seconds) for the above initial conditions and parameters of the potential shows that inflation in fact occurs up to about $10^{-32} \mathrm{~s}$ and the scale factor grows for about $10^{42}$ orders of magnitude, which corresponds to about 97 e-foldings, calculated as $N=\ln a(t) / a\left(t_{i}\right)$ and indicated on the vertical axis on the right. After that, by supposing the Elko field transfer part of its energy to ordinary matter, the universe evolves as $a(t) \sim t^{1 / 2}$ in a radiation-dominated phase, which is indicated by the straight dashed line in the logarithm scale. After that, once radiation finishes its dominance, the rest of the Elko field that was also evolving together with radiation acts again as a dark matter with null pressure and $a(t) \sim t^{2 / 3}$. The evolution follows up to present days with the scale factor of about 1, our normalization for the present day, in good agreement with the previous estimate from [1] of an increase of about $10^{74}$ for standard model.
Figure 2 shows the numerical analysis for the evolution of the energy density $\rho(t)$ from (6), shown as a solid line. It can be seen that during the inflationary phase (from $t \sim 10^{-35} \mathrm{~s}$ to $10^{-32} \mathrm{~s}$ ) the energy density is nearly constant, about $10^{155} \mathrm{~s}^{-4} \simeq 10^{58} \mathrm{GeV}^{4} \simeq$ $10^{75} \mathrm{~g} / \mathrm{cm}^{3}$. The inflation finishes with an energy density of about $10^{149} \mathrm{~s}^{-4} \simeq 10^{52} \mathrm{GeV}^{4} \simeq 10^{69} \mathrm{~g} / \mathrm{cm}^{3}$, in good agreement with the standard model [1] of about $10^{69} \mathrm{~g} / \mathrm{cm}^{3}$. After $t \simeq 10^{-30} \mathrm{~s}$ (dashed line) the evolution corresponds to the radiation energy density of the form $\rho_{r}=\rho_{r 0} a(t)^{-4}$, showing the reduction of the radiation energy density up to $10^{11} \mathrm{~s}$, where matter and radiation are in thermal equilibrium. At this stage the energy density is of about $10^{-35} \mathrm{GeV}^{4} \simeq 10^{-18} \mathrm{~g} / \mathrm{cm}^{3}$. After that the evolution follows the matter energy density (dotted line) as $\rho_{m}=\rho_{m 0} a(t)^{-3}$ up to present days, where the energy density is about $10^{50} \mathrm{~s}^{-4} \simeq 10^{-47} \mathrm{GeV}^{4} \simeq 10^{-29} \mathrm{~g} / \mathrm{cm}^{3}$, exactly the expected value for the critical energy density today. The energy density at all transitions phase are in good agreement with the standard model.

Figure 3(a) shows the numerical results for the decaying of the Elko field $\phi(t)$ from its initial value $\phi_{i}=1.75 m_{p l}$ with time. Before reaching the bottom of the potential the field oscillates for a long time around its minimal value. Figure 3(b) shows the numerical analysis of the equation-of-state parameter $\omega \equiv p(\phi) / \rho(\phi)$ obtained with (6) and (7), which indicates that $\omega$ starts from -1 during inflation and then begins to oscillate around $\omega=0$ for the rest of its evolution. This confirms that the Elko field satisfies a dust equation-of-state type, exactly as desired for a dark-matter fluid. Thus, the Elko field acts as a dark matter after the radiation-dominated era, up to the rest of the evolution of the universe. Also, having nearly null kinetic energy the Elko particles could be 


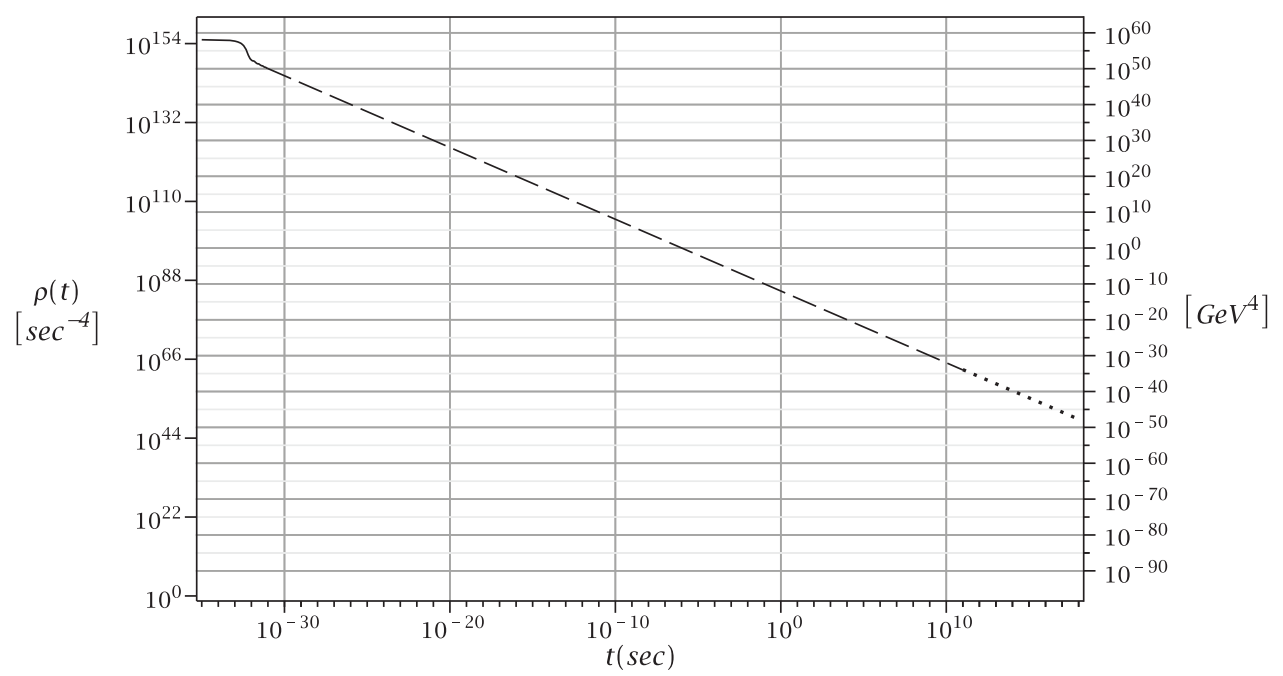

Fig. 2: Numerical result for the evolution of the energy density (6) of the Elko field with time. The unit of the vertical scale on the left is $\left(\mathrm{s}^{-4}\right)$ and on the right it is $\mathrm{GeV}^{4}$. During the inflationary phase the energy density is nearly constant, about $10^{155} \mathrm{~s}^{-4} \simeq$ $10^{58} \mathrm{GeV}^{4} \simeq 10^{75} \mathrm{~g} / \mathrm{cm}^{3}$. The inflation finishes at $10^{-32} \mathrm{~s}$ with an energy density of about $10^{149} \mathrm{~s}^{-4} \simeq 10^{52} \mathrm{GeV}^{4} \simeq 10^{69} \mathrm{~g} / \mathrm{cm}^{3}$. Up to $t \simeq 10^{-30} \mathrm{~s}$ (solid line) the figure was obtained numerically using the solution for $\phi(t)$ into $(6)$. After $t \simeq 10^{-30} \mathrm{~s}($ dashed line) the evolution corresponds to the radiation energy density of the form $\rho_{r}=\rho_{r 0} a(t)^{-4}$, with $\rho_{r 0}=4 \times 10^{39} \mathrm{~s}^{-4}$, showing the reduction of the radiation energy density up to $10^{11} \mathrm{~s}$, where matter and radiation are in equilibrium, with an energy density of about $10^{-35} \mathrm{GeV}^{4} \simeq 10^{-18} \mathrm{~g} / \mathrm{cm}^{3}$. After that, the evolution follows the matter energy density $($ dotted line $)$ as $\rho_{m}=\rho_{m 0} a(t)^{-3}$, with $\rho_{m 0}=3.5 \times 10^{35} \mathrm{~s}^{-4}$, up to present days, where the energy density is about $10^{51} \mathrm{~s}^{-4} \simeq 10^{-46} \mathrm{GeV}^{4} \simeq 10^{-29} \mathrm{~g} / \mathrm{cm}^{3}$.

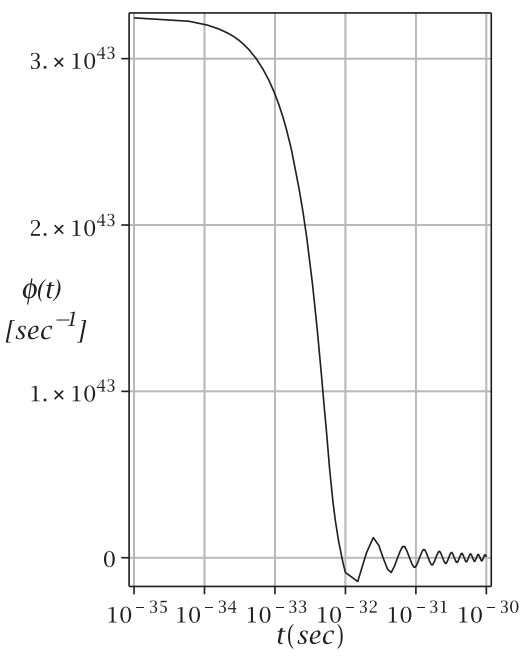

(a)

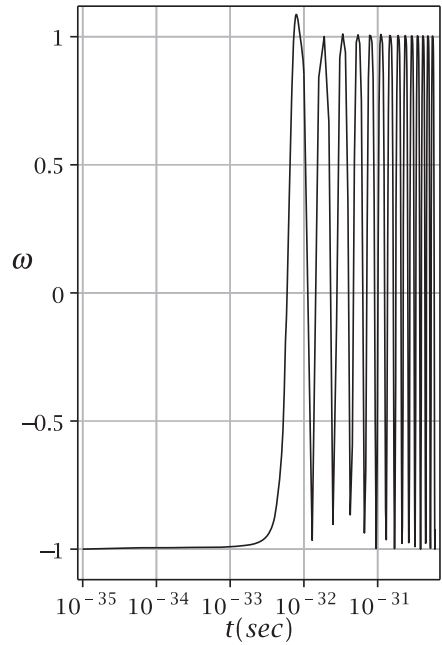

(b)

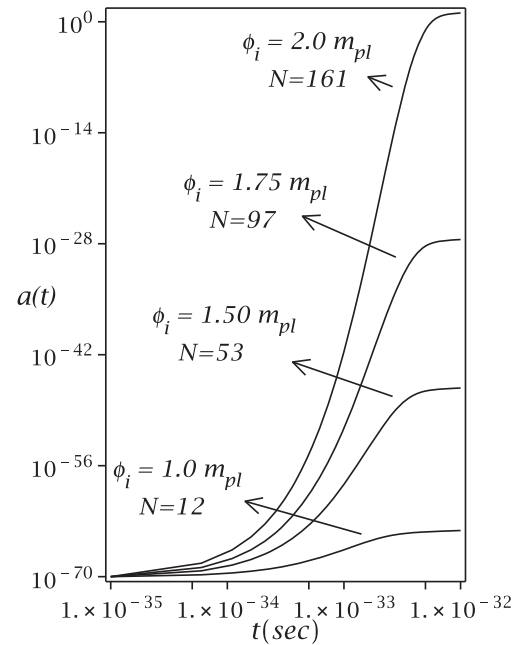

(c)

Fig. 3: (a) Evolution of the Elko field $\phi(t)$ to its minimal value showing its oscillation with time while rolling down to the bottom of the potential. (b) Evolution of the equation of state parameter $\omega=p / \rho$ with time, showing its oscillation around 0 after it starts at $\omega=-1$. (c) Detailed view of the inflationary phase and its strong dependence on the initial value $\phi_{i}$. The number $N$ of e-foldings is also presented for each case.

attracted to other local potentials, initiating the growth of small structures, such as dark-matter halos. Remember that it is one of the main characteristics of the Elko field, a natural candidate to describe dark-matter particles. Figure 3(c) shows in detail what happens during the inflationary phase for different initial conditions of $\phi$, indicating a strong dependence on the initial value $\phi_{i}$ of the field. The other parameters are the same of the previous analysis. It is possible to clearly see that the number of e-foldings of the inflation is strongly dependent on the ini- tial condition for the field. This is a kind of fine tuning for the model and shows that inflation occurs when the field decreases below the Planck mass scale.

Concluding remarks. - The numerical analysis of the coupled system of equations concerning a homogeneous and isotropic distribution of Elko fields filling the whole universe was performed. The potential under which the Elko field slows down is a quadratic mass term plus a quartic self-interaction. It was found that the evolution 
for the scale factor reproduces the expected exponential growth during the inflationary phase up to about $10^{-32} \mathrm{~s}$, with the desired number of e-foldings. The energy density after inflation is also in agreement with standard model. After inflation ends, by supposing a partial energy transfer from the Elko field to radiation, the values for the scale factor and energy density serve as initial conditions for the radiation phase. Surprisingly the evolution of the type $t^{1 / 2}$ for the scale factor and $a(t)^{-4}$ for the energy density leads the universe to the expected value for the energy density at $10^{11} \mathrm{~s}$, where matter and radiation are in equilibrium. Being the Elko field of matter type, with null pressure, it dominates again, giving rise to the dark-matter phase in the universe, evolving as $t^{2 / 3}$ for the scale factor and $a(t)^{-3}$ for the energy density. Again, the present-day value for the energy density is obtained in agreement with the standard model, and the total growth for the scale factor is about $10^{70}$, also in agreement with the standard model.

In this analysis the mass of the Elko field was taken as $1.0 \mathrm{GeV}$ and its effects during the inflationary phase is not important, thus the mass of the Elko field must be estimated by others observational constraints, such as formation of structures, for instance. We have verified that an upper limit to the mass of $m \ll 10^{-10} m_{p l} \sim 10^{9} \mathrm{GeV}$ can be established if we want to keep the initial conditions before each epoch according to the standard model values. Such value is in agreement with modern estimates [27]. It was also obtained that the number of e-foldings during the inflationary phase is strongly dependent on the initial value of the field, here taken as $\phi_{i}>m_{p l}$, as occurs in chaotic inflationary models. Inflation occurs exactly when the field goes from $\phi_{i} \sim m_{p l}$ to zero. As the field decays to the bottom of its potential it starts to oscillate and its amplitude diminishes with time.

As already pointed out previously, a similar analysis just for the inflationary phase has been done in [23], using a phenomenological symmetry-broken potential. The expected evolution as matter type after inflation has been obtained just by extrapolating the obtained curve and the effective mass estimated is strongly dependent on the parameters of the symmetry-broken potential. Here both evolutions as radiation and matter type were obtained using their expected evolution laws and initial conditions generated after inflation, showing a better agreement with the standard model. Also, the mass used here for the field is a free parameter, which can be constrained by observations, since the inflationary phase is driven just by the quartic self-interaction term.

Although the exact analysis of the system of equations has been done only for a short time interval ${ }^{5}$, the initial conditions generated after inflation for the scale factor and the energy density correctly lead to the expected values for the subsequent evolution. In this sense we can affirm that the Elko field drives all the evolution of the universe,

\footnotetext{
${ }^{5}$ The numerical analysis for the oscillatory behaviour of $\omega$ was verified from $10^{-35} \mathrm{~s}$ up to $10^{-20} \mathrm{~s}$.
}

including its action as dark matter in the last phase of evolution.

The Elko field considered here is just a classical field. As a final remark on the inflation driven by the Elko field, maybe we could interpret the inflationary expansion as a consequence of a kind of Pauli exclusion principle or degeneracy pressure effect if the field is considered as a quantum one. When the fermionic quantum Elko field rolls down to the bottom of the potential, trying to occupy its minimal energy state, the degeneracy pressure acts expanding the whole system in order to separate the particles, once they cannot occupy the same fundamental state. Such interpretation is not possible when the inflaton field is of bosonic type. Also, being a quantum field, its zero-point energy could act as a cosmological constant at the present time, explaining also the recent cosmic acceleration. Such possible quantum interpretation of the field for both inflation and recent cosmic acceleration driven by a mass-dimension-one fermion field deserves further investigations.

$* * *$

SHP is grateful to CNPq - Conselho Nacional de Desenvolvimento Científico e Tecnológico, Brazilian research agency, for financial support, grants Nos. 304297/2015-1 and 400924/2016-1. We would like to thank Prof. J. M. HofF DA Silva for valuable discussions. RFLH acknowledges financial support from CNPq (No. 303734/2014-0).

\section{REFERENCES}

[1] Kolb E. W. and Turner M. S., The Early Universe (Westview Press, USA) 1990.

[2] Weinberg S., Cosmology (Oxford University Press, Oxford, UK) 2008.

[3] Liddle A. R. and Lyth D. H., Cosmological Inflation and Large-scale Structure (Cambridge University Press) 2000.

[4] Linde A. D., in Post-Planck Cosmology, Lecture Notes of the Les Houches Summer School, edited by DefFaYeT C., Peter P., Wandelt B., Zaldarriaga M. and Cugliandolo L. F., Vol. 100 (Oxford Scholarship Online) 2013, pp. 231-316, arXiv:1402.0526 [hep-th].

[5] Planck Collaboration (Ade P. A. R. et al.), Astron. Astrophys., 571 (2014) A22.

[6] Ahluwalia-Khalilova D. V. and Grumiller D., Phys. Rev. D, 72 (2005) 067701.

[7] Ahluwalia-Khalilova D. V. and Grumiller D., JCAP, 07 (2005) 012.

[8] Ahlumalia D. V., Lee C.-Y. and Schritt D., Phys. Rev. D, 83 (2011) 065017.

[9] Ahluwalia D. V., Adv. Appl. Clifford Algebras, 27 (2017) 2247

[10] Ahluwalia D. V., EPL, 118 (2017) 60001.

[11] Bueno Rogrio R. J. and Hoff da Silva J. M., EPL, 118 (2017) 10003.

[12] Alves A., Dias M., De Campos F., Duarte L. and Hoff DA Silva J. M., arXiv:1712.05180 [hep-ph] (2017). 
[13] Fabbri L., Phys. Lett. B, 704 (2011) 255.

[14] Boenmer C. G., Phys. Rev. D, 77 (2008) 123535.

[15] Boehmer C. G., Burnett J., Mota D. F. and Shaw D. J., JHEP, 07 (2010) 053.

[16] BAsak A. and Bhatt J. R., JCAP, 06 (2011) 011.

[17] Sadjadi H. M., Gen. Relativ. Gravit., 44 (2012) 2329.

[18] Kouwn S., Lee J., Lee T. H. and Oh P., Mod. Phys. Lett. A, 28 (2013) 1350121.

[19] Pereira S. H., dos Santos Souza A. P., Hoff DA Silva J. M. and Jesus J. F., JCAP, 01 (2017) 055.

[20] Pereira S. H., dos Santos Souza A. P. and Hoff da Silva J. M., JCAP, 08 (2014) 020.
[21] Hoff da Silva J. M. and Pereira S. H., JCAP, 03 (2014) 009.

[22] dos Santos Souza A. P., Pereira S. H. and Jesus J. F., Eur. Phys. J. C, 75 (2015) 36.

[23] Pereira S. H. and Guimarães T. M., JCAP, 09 (2017) 038.

[24] Dias M., De Campos F. and Hoff da Silva J. M., Phys. Lett. B, 706 (2012) 352.

[25] Alves A., De Campos F., Dias M. and Hoff Da Silva J. M., Int. J. Mod. Phys. A, 30 (2015) 1550006.

[26] Linde A. D., Phys. Lett. B, 129 (1983) 177.

[27] Fukuyama T., Kikuchi T. and Osaka T., JCAP, 06 (2005) 005. 